\title{
PELETIZAÇÃo DE SEMENTES DE SORGO COM CALCÁRIO E COM TERMOFOSFATO E SUA INFLUÊNCIA NA ABSORÇÃO DE FÓSFORO E DE POTÁSSIO(1)
}

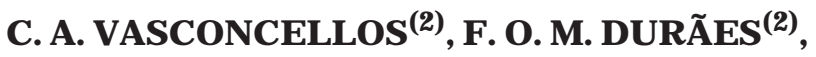 \\ R.A. RIBEIRO ${ }^{(3)} \&$ P. C. MAGALHÃE $S^{(2)}$
}

\begin{abstract}
RESUMO
O trabalho foi realizado com o objetivo de avaliar a influência da peletização de sementes de três cultivares de sorgo (Sorghum bicolor (L.) Moench), cv. BRS 701, BRS 304 e BRS 601, com calcário e com termofosfato magnesiano, na absorção de $\mathbf{P}$ e de $\mathrm{K}$. Sementes peletizadas e não peletizadas foram colocadas para germi nar, por oito dias, em temperatura ambiente. Após este período, oito plântulas com crescimento uniforme (parte aérea e raiz) foram distribuídas em vasos que continham solução nutritiva completa de Hoagland. Após 20 dias, iniciou-se a determinação da absorção de fósforo e de potássio por meio da depleção desses nutrientes na solução nutritiva. Os resultados permitiram concluir que o tipo de revestimento das sementes influenciou, de modo distinto, o crescimento de cada cultivar de sorgo. A absorção de $\mathrm{P}$ e de $\mathrm{K}$ e o total absorvido também foram influenciados pelo revestimento das sementes em virtude do aumento no crescimento inicial das plântulas. De modo geral, independentemente do tipo de revestimento das sementes, houve maior absorção de fósforo e de potássio quando as sementes foram peletizadas. A taxa de absorção de fósforo aumentou com o peso da matéria seca do sistema radicular e com o peso da matéria seca da parte aérea, mas, neste caso, até o limite de $3,5 \mu \mathrm{mol}$ planta-1 $\mathrm{h}^{-1}$ de P. Taxas de absorção aci ma deste valor não estiveram associadas ao aumento do peso da matéria seca da parte aérea.
\end{abstract}

Termos para indexação: Sorghum bicolor, absorção iônica, solução nutritiva.

(1) Recebido para publicação em abril de 1999 e aprovado em maio de 2000.

(2) Engenheiro-Agrônomo da Embrapa Milho e Sorgo (CNPMS). Caixa Postal 151, CEP 35701-970 Sete Lagoas (MG).

(3) Estudante de Biologia, Embrapa Milho e Sorgo (CNPMS). Bolsista do CNPq. 


\author{
SUMMARY: INFLUENCE OF SORGHUM SEED PELLETING WITH LIME \\ AND WITH THERMOPHOSPHATE ON PHOSPHORUS AND \\ POTASSIUM ABSORPTION
}

\begin{abstract}
Theaim of this work was to evaluatetheinfluence of seed pelleting with limeand with 'Magnesian Thermophosphate' on ionic absorption of three sorghum genotypes (Sorghum bicolor (L.) Moench), cv BRS 701, BRS 304 e BRS 601. Pelletized and non-pell etized seeds weregermi nated during eight days at room temperature. After this period, eight plants with uni form root and shoot devel opment, from each treatment, were transferred to a Hoagland solution. Phosphorus and potassium absorption parameters weredetermined after 20 days. Phosphorus and potassium uptakes varied among sorghum genotypes and wereinfluenced by the compound used in the pelletization, which affected initial plant growth. Phosphorus absorption rateshowed positivecorrelation with thedry matter of the root system and with shoot dry matter up to $3.5 \mu \mathrm{mol} P$ plant ${ }^{-1} \mathrm{~h}^{-1}$ of $\mathrm{P}$. Absorption rates higher than this value did not show significant correlation with shoot dry matter.
\end{abstract}

Index terms: Sorghum bicolor, ionic absorption, nutrient solution.

\section{INTRODUÇÃO}

Os solos da região tropical são, na sua maioria, provenientes de rochas com baixos teores de fósforo, altamente intemperizados, com condições favoráveis à fixação de fósforo e, conseqüentemente, à redução na sua disponibilidade para as plantas (Parfitt, 1978).

De modo geral, a proximidade dos fertilizantes na regiãodeabsorção denutrientes pelo sistema radicular diminui a influência do solo na disponibilidade de nutrientes, favorecendo o desenvolvimento adequado das plântulas ea mel hor eficiência no aproveitamento dos fertilizantes fosfatados (Anghinoni, 1992).

Neste contexto, a prática de peletização de sementes tem-se mostrado viável, especialmente para o estabelecimento da cultura do sorgo em sucessão a soja, quando a velocidade de semeadura, emergência, crescimento e desenvolvimento das plântulas faz-se prioritária para melhor utilização do período chuvoso (Magal hães et al., 1994).

Na maximização da produtividade de sorgo, tem sido comum a semeadura a lanço imediatamente após o início da queda das folhas da soja. Nesta prática, o aumento da massa das sementes, decorrente da peletização, facilita sua distribuição, aumenta a velocidade do crescimento e do desenvolvimento inicial das plântulas e contribui para melhorar o aproveitamento das condições hídricas (Magal hães et al., 1994).

Outro ponto a ser considerado é a interação de nutrientes. Wilkinson \& Duncan (1993), por exemplo, determinaram que o magnésio favorece a absorção diferencial decál cio por cultivares de sorgo. Crocomo \& Malavolta (1964) e Lourenço (1967) relataram que a absorção e a translocação de fósforo em raízes destacadas de cevada foram estimuladas pela presença de quantidades crescentes de magnésio.
Sherrell et al. (1964) encontraram que o fósforo aplicado às sementes de aveia favoreceu a absorção iônica, em comparação com outras formas de aplicação. Garg \& Welch (1967) encontraram resultados semel hantes para milho. Miller et al. (1971) determinaram quefertilizantes junto às sementes demilho promoveram aumento de $36 \%$ na matéria seca nas plantas com 36 dias, decréscimo de 1,2 dia para o pendoamento e aumento de 2,9\% na produção de grãos.

Poucas são as informações sobre o efeito da peletização das sementes na nutrição das plantas, principalmente sobre sua influência na absorção iônica. O objetivo deste trabalho foi avaliar a influência da peletização das sementes de três cultivares de sorgo na absorção de $\mathrm{P}$ e de $\mathrm{K}$.

\section{MATERIAL E MÉTODOS}

O trabal ho foi realizado em condições de casa de vegetação, no CentroNacional de Pesquisas de Milho e Sorgo, situado no município de Sete Lagoas (MG), altitude de $732 \mathrm{~m}$, latitude de $19^{\circ} 28^{\prime} \mathrm{S}$ e longitude de $45^{\circ}$ 15'W (Avelar, 1982).

Sementes de três cultivares de sorgo (BRS 701 , BRS 304 eBRS 601) foram pel etizadas com cal cário cal cítico (PRNT $=75,9 \% ; \mathrm{PN}=95 \%$, resíduo insolúvel =9,8\%, $\mathrm{CaO}=47,7 \% ; \mathrm{MgO}=1,9 \%)$ e com termofosfato magnesiano $\left(16 \%\right.$ de $\mathrm{P}_{2} \mathrm{O}_{5}$ solúvel em ácido cítrico, $9 \%$ de $\mathrm{Mg}$ e $20 \%$ de $\mathrm{Ca}$ ). As sementes foram umedeci das com uma solução de goma-arábica a $5 \%$, submetidas a movimentos circulares em contato com a substância que as envolveriam, conforme procedimento descrito por Norris et al. (1970), e colocadas para secar em temperatura ambiente. As quantidades de calcário e do termofosfato magnesiano, aderidas a 50 sementes de cada cultivar, encontram-se no quadro 1. 
Quadro 1. Massa de 50 sementes de sorgo peletizadas com calcário e termofosfato magnesiano

\begin{tabular}{llll}
\hline \multirow{2}{*}{ Cultivar } & \multicolumn{2}{c}{ Tipo de pelete(1) } & Sem pelete \\
\cline { 2 - 3 } & $\begin{array}{c}\text { Termofosfato } \\
\text { magnesiano }\end{array}$ & Calcário & \\
\hline & & & \\
BRS 701 & $1,46(0,06)$ & $1,55(0,15)$ & 1,40 \\
BRS 304 & $2,39(0,09)$ & $2,42(0,12)$ & 2,30 \\
BRS 601 & $1,61(0,25)$ & $1,44(0,08)$ & 1,36
\end{tabular}

(1) O número entre parênteses refere-se à quantidade de termofosfato magnesiano ou de cal cário, aderida a 50 sementes.

Posteriormente, as sementes pel etizadas ou não foram colocadas para germinar em papel-toalha e umedecidas com água destilada, durante oito dias, em temperatura ambiente $\left(25^{\circ} \mathrm{C} \pm 3\right)$, segundo Magnavaca (1982). A pós este período, oito plântulas com crescimento uniforme (parteaérea eraiz) foram distribuídas e mantidas, por 20 dias, em vasos de 9,89 L com solução nutritiva completa, continuamentearejadas, com as seguintes concentrações, em

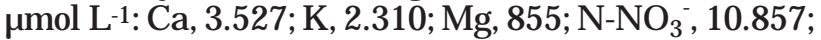
$\mathrm{N}-\mathrm{NH}_{4}{ }^{+}, 1.300 ; \mathrm{P}, 45 ; \mathrm{S}, 587 ; \mathrm{B}, 25 ; \mathrm{Fe}, 77 ; \mathrm{Mn}, 9,1$; Cu, 0,63; Mo 0,83; Zn, 2,29, HEDTA, 75 (usado para preparar o FeHEDTA (hidroxietilenodiaminetriacetato de ferro); $\mathrm{Na}, 1,74$ (Magnavaca, 1982). $\mathrm{O}$ pH da solução nutritiva foi monitorado periodicamente sem, contudo, efetuar-se sua correção, procurando determinar apenas o efeito da pel etização. O pH inicial foi ajustado em 4,8, conforme proposto por Clark (1982).

Após este período, iniciaram-se etapas para determinar a depleção de potássio e de fósforo, conforme descrito por Ruiz (1985), usando quatro repetições. As plantas foram mantidas, por $24 \mathrm{~h}$, em solução nutritiva completa menos o fósforo ou 0 potássio.

Ao final deste período, o elemento faltante foi reposto à respectiva solução na concentração de $26,1 \mu \mathrm{mol} \mathrm{L}^{-1}$ de $\mathrm{P}$ e 0,54 mmol L-1 de $\mathrm{K}$ no tempo inicial da depleção. Alíquotas de $5 \mathrm{~mL}$ foram tomadas a interval os constantes de 30', durante 5 h e 30', para estimar a exaustão de cada elemento na solução nutritiva. A curva de depleção de potássio foi obtida com oito plantas por vaso (1.240 mL/planta); a de fósforo, com seis ( $1.650 \mathrm{~mL} / \mathrm{planta})$. Com este procedimento, procurou-se obter o comportamento estacionário da curva de absor ção e, ou, de depl eção adequado à aplicação do modelo cinético (Hagen \& Hopkins, 1955; Alves, 1984; Ruiz, 1985).

Posteriormente, as plantas foram separadas em parte aérea e sistema radicular, secas a $75^{\circ} \mathrm{C}$, por
$48 \mathrm{~h}$, e pesadas. O potássio na solução foi determinado por fotometria de chama e ofósforo pelo método de Murphy \& Riley (1962), adaptado por Braga \& Defelipo (1974). As diferenças entre os coeficientes (intercepto e decl ividade) das equações ajustadas foram avaliadas pel o testet a $5 \%$, segundo Stell \& Torrie (1960). O decréscimo da concentração do el emento na solução nutritiva representou sua absorção pelo sistema radicular das plantas, como um todo.

As taxas de depleção (TD) de $\mathrm{K}$ e de $\mathrm{P}$ foram calculadas da seguinte forma:

$$
\mathrm{TD}=\mathrm{CV} / \mathrm{PAn}
$$

sendo

C = coeficienteangular da equação ajustada entre a concentração ( $\mathrm{mg} \mathrm{mL}^{-1}$ ) e o tempo;

$\mathrm{V}=$ volume do vaso $(\mathrm{mL})$;

PA = peso atômico do el emento em estudo;

$\mathrm{n}=$ número de plantas por vaso

A análise estatística dos dados obedeceu ao delineamento de blocos ao acaso com parcelas subdivididas com quatro repetições, tendo-se o cultivar e o tipo de pel ete nas parcelas e o tempo de amostragem nas subparcelas. As análises estatísticas foram efetuadas pelo uso do programa MSTAC (1991).

\section{RESULTADOS E DISCUSSÃO}

O peso de matéria seca de raízes e parte aérea das plantas usadas para a estimativa da depleção defósforo e de potássio encontra-se no quadro 2. Tais dados demonstram que os diferentes tipos de revestimentos influenciaram o crescimento dessas plantas. No caso da depleção de potássio, por exemplo, independentemente do cultivar, o revestimento com calcário favoreceu maiores pesos da parte aérea e dosistema radicular. O revestimentocom termofosfato promoveu maiores aumentos da parte aérea e das raízes apenas no cultivar BRS 601.

No caso da depleção de fósforo, observaram-se aumentos, em geral, significativos da parte aérea e do sistema radicular, tanto para o revestimento com o termofosfato como com calcário, exceto para o cultivar BRS 304.

A anál ise de variância para a depl eção de potássio (C.V. $=4 \%$ ) determinou diferenças significativas $(p<0,01$ ) entre o tempo de amostragem, cultivar e tipo de revestimento das sementes (peletes). As equações ajustadas entre a concentração de $\mathrm{K}$ na solução nutritiva e o tempo de amostragem encontram-se no quadro 3. Com base nesses dados, calculou-se a taxa de depl eção de K. 
Quadro 2. Peso da parte aérea e das raízes das plantas de sorgo usadas na depleção de P e K influenciado pelo tipo de revestimento das sementes

\begin{tabular}{|c|c|c|c|c|c|}
\hline \multirow{2}{*}{ Cultivar } & \multirow{2}{*}{ Tipo de revestimento } & \multicolumn{2}{|c|}{ Depleção de K } & \multicolumn{2}{|c|}{ Depleção de P } \\
\hline & & Parte aérea & Raiz & Parte aérea & Raiz \\
\hline & & & - & ta & 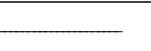 \\
\hline BRS 701 & $\begin{array}{l}\text { Testemunha(1) } \\
\text { Magnesiano } \\
\text { Calcário }\end{array}$ & $\begin{array}{l}0,16 \mathrm{~cd} \\
0,15 \mathrm{~d} \\
0,31 \mathrm{abc}\end{array}$ & $\begin{array}{l}0,06 \mathrm{bc} \\
0,04 \mathrm{c} \\
0,11 \mathrm{ab}\end{array}$ & $\begin{array}{l}0,31 \mathrm{c} \\
0,48 \mathrm{bc} \\
0,62 \mathrm{ab}\end{array}$ & $\begin{array}{l}0,13 \mathrm{~d} \\
0,18 \mathrm{~cd} \\
0,21 \mathrm{bcd}\end{array}$ \\
\hline BRS 304 & $\begin{array}{l}\text { Testemunha } \\
\text { Magnesiano } \\
\text { Calcário }\end{array}$ & $\begin{array}{l}0,27 \mathrm{abcd} \\
0,29 \mathrm{abc} \\
0,36 \mathrm{ab}\end{array}$ & $\begin{array}{l}0,10 a b c \\
0,10 a b c \\
0,16 a\end{array}$ & $\begin{array}{l}0,61 a b \\
0,62 a b \\
0,62 a b\end{array}$ & $\begin{array}{l}0,25 \mathrm{abcd} \\
0,28 \mathrm{abc} \\
0,32 \mathrm{ab}\end{array}$ \\
\hline BRS 601 & $\begin{array}{l}\text { Testemunha } \\
\text { Magnesiano } \\
\text { Calcário }\end{array}$ & $\begin{array}{l}0,23 \mathrm{bcd} \\
0,35 \mathrm{ab} \\
0,41 \mathrm{a}\end{array}$ & $\begin{array}{l}0,10 a b c \\
0,13 a b \\
0,14 a\end{array}$ & $\begin{array}{l}0,47 \mathrm{bc} \\
0,60 \mathrm{ab} \\
0,70 \mathrm{a}\end{array}$ & $\begin{array}{l}0,23 \mathrm{bcd} \\
0,34 \mathrm{ab} \\
0,36 \mathrm{a}\end{array}$ \\
\hline dms g/planta & & 0,13 & 0,06 & 0,19 & 0,13 \\
\hline
\end{tabular}

(1) Médias seguidas pelas mesmas letras, dentro de uma mesma coluna, não apresentam diferenças significativas pelo Teste de Tukey a $5 \%$.

Conforme Ruiz (1985) e Castells et al. (1985), o valor de b (declividade da reta) é equivalente a V máx, quando associado à depleção por unidade de raiz. Optou-se, contudo, por expressar os resultados como taxa de depleção ( $\mu$ mol planta-1 $\mathrm{h}^{-1}$ ) e avaliar a variabilidade deste fator de acordo com os pesos da parte aérea e do sistema radicular (Figura 1).

Dentreos cultivares, as maiores taxas de depleção de potássio foram observadas para cultivar BRS 701 e as menores para BRS 304. Todos os cultivares apresentaram, de modo geral, aumentos na taxa de depleção de potássio com o revestimento das sementes. O cultivar BRS 701 apresentou maior aumento na depleção, quando o revestimento foi efetuado com calcário, e os cultivares BRS 304 e BRS 601, quando o revestimento foi efetuado com termofosfato magnesiano (Quadro 3). Magal hães et al. (1994) demonstraram, de modo análogo, que a pel etização de sementes influencia o crescimento e o desenvolvimento vegetativo do sorgo, sendo a magnitude do efeito dependente dos cultivares e do tipo de revestimento utilizado.

Os cultivares BRS 701 e BRS 304 apresentaram aumentos da taxa de depleção de potássio com os dois tipos de revestimento de sementes; o cultivar BRS 601 apenas com o termofosfato magnesiano (Quadro 3).

O teste do coeficiente angular das regressões ajustadas entre otempoea absorção deK demonstrou haver diferenças apenas para as declividades entre os cultivares eo tipo de revestimento. No revestimento com cal cário, o cultivar BRS 601 apresentou comportamento semel hante ao do tratamento testemunha.

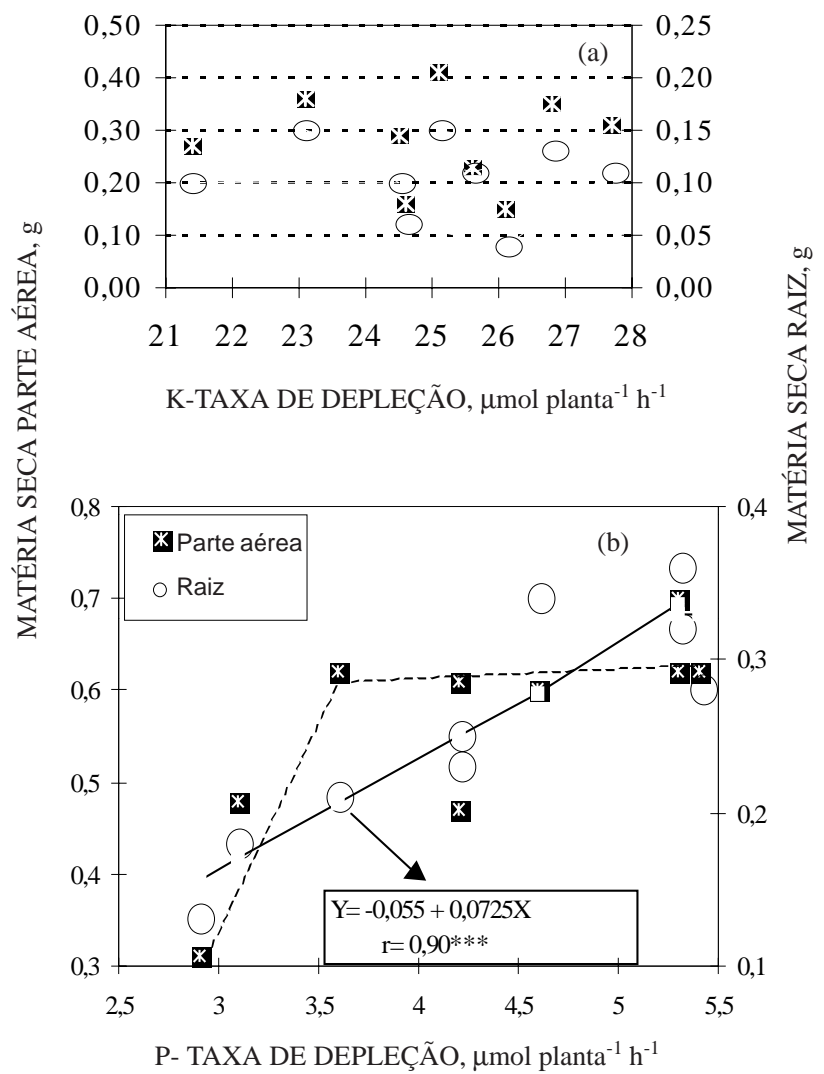

Figura 1. Relação entre as taxas de depleção de potássio e de fósforo por três cultivares de sorgo, cujas sementes foram peletizadas ou não com calcário e com termofosfato magnesiano e suas respectivas massas da matéria seca da parte aérea e das raízes. 
Quadro 3. Concentração de potássio $\left(Y, \mu \mathrm{g} \mathrm{mL}^{-1}\right)$ na solução nutritiva, considerando tempo de exaustão ( $X$, horas), três cultivares de sorgo, sementes peletizadas com calcário e termofosfato magnesiano ou não, e sua respectiva taxa de depleção de $K$

\begin{tabular}{|c|c|c|c|c|}
\hline Cultivar & Tipo de revestimento & E quação & $\mathbf{R}^{2}$ & Taxa de depleção \\
\hline & & & $\%$ & $\mu m o l$ planta-1 $\mathrm{h}^{-1}$ \\
\hline \multirow{3}{*}{ BRS 701} & Testemunha & $Y=21,06-0,777 X$ & $92 * * *$ & 24,6 \\
\hline & Magnesiano & $Y=21,24-0,825 X$ & $84 * * *$ & 26,1 \\
\hline & Calcário & $Y=21,20-0,877 X$ & $92 * * *$ & 27,7 \\
\hline \multirow{3}{*}{ BRS 304} & Testemunha & $Y=20,95-0,677 X$ & $98 * * *$ & 21,4 \\
\hline & Magnesiano & $Y=21,34-0,776 X$ & $98 * * *$ & 24,5 \\
\hline & Calcário & $Y=21,27-0,729 X$ & $98 * * *$ & 23,1 \\
\hline \multirow{3}{*}{ BRS 601} & Testemunha & $Y=21,50-0,808 X$ & $97 * * *$ & 25,6 \\
\hline & Magnesiano & $Y=21,30-0,848 X$ & $97 * * *$ & 26,8 \\
\hline & Calcário & $Y=21,50-0,794 X$ & $97 * * *$ & 25,1 \\
\hline
\end{tabular}

*** Significativo a $1 \%$.

A análise de variância para a depleção de fósforo (C.V. 14,2\%) demonstrou haver diferenças significativas entre os cultivares de sorgo e tipo de revestimento de sementes. As maiores taxas de depleção foram obtidas para o cultivar BRS 304 eas menores para o BRS 701 (Quadro 4).

O tipo de revestimento das sementes influenciou, de modo distinto, cada cultivar de sorgo. O cal cário favoreceu maiores absorções de fósforo no BRS 701 e no BRS 601. O cultivar BRS 304 não apresentou diferenças entre o revestimento de calcário e o termofosfato magnesiano (Quadro 4).

A taxa de depleção de $P$ foi também influenciada pelo revestimento das sementes, tendo o revestimento com calcário favorecido maiores depleções. Faquin et al. (1990) também determinaram que a vel ocidade máxima da absorção de fósforo foi influenciada pelo estado nutricional das plantas de soja, determinado por diferentes níveis de adubação.

Essas diferenças na absorção iônica poderiam, também, refletir alterações no pH da solução nutritiva. Todavia, a análise de variância (C.V. 3,3\%) demonstrou haver decréscimos significativos apenas com o tempo de amostragem e com o cultivar. O tipo de revestimento não alterou o pH da solução nutritiva. Ao final do experimento, o pH da solução apresentou os seguintes valores: cultivar BRS 701pH 4,0b, BRS 304- pH 4,1b e BRS 601 pH 4,3a. Portanto, pelopH da soluçãonutritiva nãofoi possível explicar as variações na absorção iônica. Sabe-se apenas que o cultivar BRS 601 favoreceu menor decréscimo de $\mathrm{pH}$ do meio de crescimento.

Houve correlação positiva e significativa ( $r=0,90^{* * *}$ ) entre o peso de matéria seca de raízes e a taxa de depleção defósforo (Figura 1). Ao consi derar o peso da matéria seca da parte aérea, a taxa de absorção de fósforo aumentou até o limite de 3,5 $\mu \mathrm{mol}$ planta-1 $\mathrm{h}^{-1}$ de P. A taxa de absorção acima deste valor apresentou variações a despeito do nãoaumento do peso de matéria seca da parte aérea.

Apesar do aumento de absorção decorrente do acréscimo do peso da matéria seca do sistema radicular, não se observou tal fato com o aumento da massa da parte aérea entre 0,3 e 0,7 g. Portanto, em termos de eficiência de absorção de fósforo, a massa da matéria seca da parte aérea pode não ser um indicador eficiente, pois taxas de absorção superiores a 3,5 $\mu \mathrm{mol}$ planta-1 $\mathrm{h}^{-1}$ deP não estiveram associadas a aumentos de peso da matéria seca.

A taxa de depleção de potássio não mostrou relação significativa com as massas do sistema radicular e da parte aérea. É provável que esses resultados estejam associados às funções metabólicas do elemento específico. Diferentemente do potássio, o fósforo exerce funções que envolvem balanço e transporte de energia (E pstein, 1975).

Independentemente do cultivar e do tipo de revestimento de sementes, as taxas de depleção de fósforo mostraram-se dependentes da massa da matéria seca de raízes, ou seja, a absorção do fósforo dependeu da quantidade do sistema radicular do cultivar de sorgo. Römer et al. (1989), entretanto, mencionaram que, para plantas detrigo com 21 dias, na presença de disponibilidade adequada de fósforo, - sistema radicular não foi importante para discriminar a eficiência de absorção e, quando em baixa disponibilidade, podeter havido adaptação por meio do aumento das raízes ou mesmo da efetividade da absor ção de fósforo por uni dade de raiz. A sel eção deplantas eficientes, portanto, poderia ser efetuada, procurando esta adaptação aliada às maiores produtividades. 
Quadro 4. Concentração de fósforo $\left(Y, \mu \mathrm{g} \mathrm{mL}^{-1}\right)$ na solução nutritiva, considerando tempo de exaustão (X, horas), três cultivares de sorgo, sementes peletizadas com calcário e termofosfato magnesiano ou não, e sua respectiva taxa de depleção de $P$

\begin{tabular}{|c|c|c|c|c|}
\hline Cultivar & Tipo de revestimento & E quação & $\mathbf{R}^{2}$ & Taxa de depleção \\
\hline & & & $\%$ & $\mu m o l$ planta-1 $\mathrm{h}^{-1}$ \\
\hline BRS 701 & $\begin{array}{l}\text { Testemunha } \\
\text { Magnesiano } \\
\text { Calcário }\end{array}$ & $\begin{array}{l}Y=0,80-0,054 X \\
Y=0,81-0,058 X \\
Y=0,80-0,068 X\end{array}$ & $\begin{array}{l}99 * * * \\
99 * * * \\
99 * * *\end{array}$ & $\begin{array}{l}2,9 \\
3,1 \\
3,6\end{array}$ \\
\hline BRS 304 & $\begin{array}{l}\text { Testemunha } \\
\text { Magnesiano } \\
\text { Calcário }\end{array}$ & $\begin{array}{l}Y=0,78-0,078 X \\
Y=0,79-0,101 X \\
Y=0,82-0,099 X\end{array}$ & $\begin{array}{l}97 * * * \\
99 * * * \\
98 * * *\end{array}$ & $\begin{array}{l}4,2 \\
5,4 \\
5,3\end{array}$ \\
\hline BRS 601 & $\begin{array}{l}\text { Testemunha } \\
\text { Magnesiano } \\
\text { Calcário }\end{array}$ & $\begin{array}{l}Y=0,83-0,078 X \\
Y=0,82-0,087 X \\
Y=0,84-0,099 X\end{array}$ & $\begin{array}{l}98^{* * *} \\
98^{* * *} \\
98^{* * *}\end{array}$ & $\begin{array}{l}4,2 \\
4,6 \\
5,3\end{array}$ \\
\hline
\end{tabular}

*** Significativo a $1 \%$.

O tipo de revestimento, influenciando o melhor crescimento inicial das plântulas, acarretou maior absorção de P. Este fato implica alterações na absorção iônica de cultivares de sorgo quando a adubação é feita próximo às sementes.

O uso de peletes em sementes de sorgo, apesar de haver interação do tipo de revestimento e o cultivar, é uma prática passível de favorecer mel hor crescimento das plântulas, principalmente quando for cultivado no período outono-inverno, em sucessão às culturas de verão, quando há necessidade de melhor aproveitamento das condições de umidade do solo.

\section{CONCLUSÕES}

1. O uso de peletes em sementes favoreceu o crescimento e o desenvolvimento inicial das plântulas de sorgo e a absorção de P e de K.

2. Independentemente dos cultivares e do tipo de revestimento de sementes, as taxas de depleção de fósforo mostraram-se diretamente proporcionais à matéria seca de raízes.

\section{LITERATURA CITADA}

ALVES, V.M.C. Efeito do alumínio sobre a cinética de absorção e translocação de fosfato sobre o crescimento e composição mineral de dois cultivares de trigo. Viçosa, Universidade Federal de Viçosa, 1984. 45p. (Tese de Mestrado)

ANGHINONI, I. Uso de fósforo pelo milho afetado pela fração de solo fertilizada com fosfato solúvel. R. Bras. Ci. Solo, 16:349-353, 1992.
AVELAR, B.C. Cinqüenta anos de observação meteorológicas de Sete Lagoas, MG. Sete Lagoas, Centro Nacional dePesquisa de Milho e Sorgo, EMBRAPA. 1982. 33p. (Boletim Agrometeorológico, 4)

BRAGA, J.M. \& DEFELIPO, B.V. Determinação espectrofotométrica de fósforo em extratos de sol o e material vegetal. R. Ceres, 21:73-85, 1974.

CASTELLS, A.J .; NEVES, J .C.L.; BARROS, N.F \& NOVAIS, R.F . Considerações referentes à determinação de parâmetros cinéticos de absorção de fósforo. R. Ceres, 32:85-91, 1985.

CLARK, R.B. Nutrient solution growth of sorghum and corn in mineral nutrition studies. J. Plant Nutr., 5:1039-1057, 1982.

CROCOMO, O.J.\& MALAVOLTA, E. The uptake of radiophosphate by barley plant as influenced by magnesium. An. ESALQ, 21:43-49, 1964.

EPSTEIN, E. Nutrição mineral das plantas: princípios e perspectivas. Rio de J aneiro, Livros Técnicos e Científicos/ São Paulo, Universidade de São Paulo, 1975. 341p.

FAQUIN, V.; MALAVOLTA, E. \& MURAOKA, T. Cinética da absorção de fosfato em soja sob a influência de micorriza vesículo-arbuscular . R. Bras. Ci. Solo, 14:41-48, 1990.

GARG, K.P. \& WELCH, L.F. Growth and phosphorus uptake by corn as influenced by phosphorus placement. Agron. J., 59:152-154, 1967.

HAGEN, C.E. \& HOPKINS, H.T. I onic species in orthophophate absorption by barley roots. Plant Physiol., 30:193-199, 1955.

LOURENÇO, S. Absorção de fósforo por raízes destacadas de cevada (Hordeum vulgare) em presença de magnésio. Piracicaba, Escola Superior de Agricultura Luiz de Queiroz, 1967. 26p. (Tese de Mestrado)

MAGALHÃES, P.C.; FERREIRA, D.M.N.; AZEVEDO, I.T.; VASCONCELLOS, C.A. \& BORBA, C.S. Efeito de pel etização na germinação e desenvol vimento de cultivares de sorgo. R. Bras. Sementes, 16:20-25, 1994. 
MAGNAVACA, R. Genetic variability and the inheritance of aluminum tolerance in maize (Zea mays, L.). Lincoln, University of Nebrasca, 1982. 135p.

MSTAT-C. Microcomputer statical program. Michigan State University, 1991.

MILLER, M.H.; BATES, T.E.; SINGH, D.\& BAWEJ A, A.S. Response of corn to small amounts of fertilizer placed with the seed. I. Greenhousestudies. Agron. J ., 63:365-368, 1971.

MURPHY, J \& \& RILEY, J .P. A modified single solution method for the determination of phosphate in natural waters. Anal. Chem. Acta, 23:31-36, 1962.

NORRIS, D.O.; LOPES, E.S. \& WEBER, D.F. Incorporação de matéria orgânica ("mulching") e aplicação de péletes de calcário (pelleting) para tratar stirpes de Rhizobium em esperimentos de campo sob condições tropicais. Pesq. Agropec. Bras., 5:129-146, 1970.

PARFITT, R.L. Anion adsorption by soils and soil materials. Adv. Agron., 30:1-50, 1978
RÖMER, W.; AUGUSTIN, J . \& SCHILLING, G. Therelationship between phosphate absorption and root length in nine wheat cultivars. In LOUGHMAN, B.C.; GASPAROKOVA, O. \& KOLET, J ., eds. Structural and functional aspects of transport in roots, Dordrecht, Kluwer Academic Publishers, 1989. p.123-125.

RUIZ, H.A. Estimativa dos parâmetros cinéticos Km e Vmáx por uma aproximação gráfico-matemática. R. Ceres, 32:7984, 1985.

SHERRELL, C.G.; KETCHESON, J.W. \& MILLER, M.H. The effect of placement and banded fertilizer on fertilizer phosphorus absorption and yield of oats in greenhouse and field experiments. Can. J. Soil Sci., 44:329-336, 1964.

STELL, R.G.D. \& TORRIE, J.H. Principles and procedures of statistics. New York, McGraw-Hill Book Company, 1960. 481p.

WILKINSON, R.E. \& DUNCAN, R.R. Magnesium influence on calcium $(45 \mathrm{Ca}+2)$ absorption by sorghum root tips. J . Plant Nutr.,16:1917- 1920, 1993. 
C. A. VASCONCELLOS et al. 